\title{
EXISTENCE OF REGULAR FINITE INVARIANT MEASURES FOR MARKOV PROCESSES
}

\section{F. DENNIS SENTILLES}

Let $S$ be a locally compact Hausdorff space and let $P(t, x, \cdot)$ be a transition function on the Borel subsets of $S$ with $P(t, x, S) \leqq a>0$ for all $t \geqq 0, x \in S$. In a recent paper V. E. Beneš [1] obtains several necessary and sufficient conditions that $P(t, x, \cdot)$ have an invariant measure $\mu$ in the space $M(S)^{+}$of strictly positive bounded regular Borel measures on $S$ in the presence of several overriding conditions on the function $P$ and the space $S$. We propose to eliminate the need for these conditions by making use of the fact that $M(S)$ is the dual of the locally convex space $C(S)$ of bounded continuous functions on $S$ with the strict topology of Buck [3] (see also [9] for a more general discussion of this topology) and replace the use of weak compactness in $M(S)$ in [1] by that of $\beta$-weak* compactness studied by Conway [4] (these two notions of compactness are studied in [8]) or equivalently, in the notation of [6, p. 32], $\sigma(M(S), C(S))$-compactness.

The referee has pointed out that our results are also an improvement of some of the results in [2], where the same author does replace weak compactness in $M(S)$ by $\sigma\left(M(S), C_{0}(S)\right)$-compactness in the presence of certain other conditions. After proving our main theorem we will discuss [2] in more detail.

Our only assumption on $P$ is that for each $t \geqq 0$ the function $\left[T_{t} f\right](x)$ $=\int_{S} f(s) P(t, x, d s)$ belongs to $C(S)$ for all $f \in C(S)$. Equivalently, the function $x \rightarrow P(t, x, \cdot)$ is a continuous function on $S$ into $M(S)$ with the $\sigma(M(S), C(S))=\sigma(M, C)$ topology, for in this topology a net $\nu_{\alpha} \rightarrow \nu$ if and only if $\int_{S} f d \nu_{\alpha} \rightarrow \int_{S} f d \nu=\langle f, \nu\rangle$ for all $f \in C(S)$. It follows from [7] that for each $\mu \in M(S)$ and Borel set $E$ the formula

$$
\left(U_{t} \mu\right)(E)=\int_{S} P(t, s, E) \mu(d s)
$$

defines a measure in $M(S)$. (An appeal to [7] also yields [2, Lemma 1].) Moreover, $\left\{U_{t}: t \geqq 0\right\}$ is a semigroup of $\sigma(M, C)$-continuous operators in $M(S)$ and $\langle g, U, \mu\rangle=\int_{S} \int_{s} g(s) P(t, x, d s) \mu(d x)$ for any bounded Borel measurable function $g$ on $S$.

We denote by $C_{0}(S)$ those functions $f \in C(S)$ such that

$$
\{s \in S:|f(s)| \geqq \epsilon\}
$$

Received by the editors July 25, 1968. 
is compact in $S$ for each $\epsilon>0$. A nonnegative Borel measurable function $g$ on $S$ will be called a moment if $\exists$ a sequence $\left\{K_{n}\right\}$ of compact subsets of $S$ with $K_{n} \subset K_{n+1}^{0}$ (the interior of $K_{n+1}$ ) such that $\lim _{n \rightarrow \infty} \sup _{x \in K_{n} \backslash K_{n+1}^{0}} g(x)=\infty$, with $g$ bounded away from zero on $\bigcup_{n=1}^{\infty} K_{n}$. If $g$ is a moment it is easy to see that $\exists \phi \in C_{0}(S)^{+}$such that $1 / g(x) \leqq \phi(x)$ for $x \in \bigcup_{n=1}^{\infty} K_{n}$.

Our main result which includes that of $[1]$ is

THEOREM. The following are equivalent:

(a) $P(t, x, \cdot)$ has an invariant measure in $M(S)+$.

(b) $\exists \mu \in M(S)^{+}$and a moment $g$ such that for $t \geqq 0 U_{t} \mu\left(S \backslash \bigcup_{n=1}^{\infty} K_{n}\right)$ $=0$ and $\sup _{t \geqq 0}\left\langle g, U_{t} \mu\right\rangle<\infty$.

(c) $\exists \mu \in M(S)^{+}, \phi \in C_{0}(S)^{+}$such that all measures $U_{t} \mu$ vanish off the nonzeroes of $\phi$ and $\sup _{t \geq 0}\left\langle 1 / \phi, U_{t} \mu\right\rangle<\infty$.

(d) $\exists \mu \in M(S)^{+}, K$ a convex $\sigma(M, C)$-compact subset of $M(S)$ such that $\left\{U_{t} \mu: t \geqq 0\right\} \subset K$.

(e) $\exists \mu \in M(S)^{+}$such that the measures $\left\{U_{t} \mu: t \geqq 0\right\}$ are uniformly countably additive.

(f) $\exists \mu \in M(S)^{+}, K a \sigma\left(M(S), M(S)^{*}\right)$ compact subset of $M(S)$ such that $\left\{U_{t} \mu: t \geqq 0\right\} \subset K$.

Proof. We will show that $(a) \Rightarrow(b) \Rightarrow(c) \Rightarrow(d) \Rightarrow(a)$ and that (a) $\Rightarrow(\mathrm{e}) \Rightarrow(\mathrm{f}) \Rightarrow(\mathrm{d})$.

(a) $\Rightarrow$ (b). If $\mu$ is the assumed regular invariant measure on the locally compact space $S$, then $\exists$ a sequence $\left\{K_{n}\right\}$ of compacta such that $K_{n} \subset K_{n+1}^{0}$ and $\mu\left(S \backslash K_{n}\right)<1 / n^{3}$. Setting $g(x)=n$ for $x \in K_{n}$ and $g(x)=1$ for $x \notin \bigcup_{n=1}^{\infty} K_{n}$ yields the proof.

(b) $\Rightarrow$ (c). We choose $\phi \in C_{0}(S)^{+}$so that $1 / g \leqq \phi$ on $\cup_{n=1}^{\infty} K_{n}$. Then $\{x: \phi(x)=0\} \subset S \backslash \cup_{n=1}^{\infty} K_{n}$ and $\left\langle 1 / \phi, U_{t} \mu\right\rangle \leqq\left\langle g, U_{t} \mu\right\rangle$ for all $t \geqq 0$.

(c) $\Rightarrow(\mathrm{d})$. By $[4$, Theorem 2.1] $\{, \mu: t \geqq 0\}$ is an equicontinuous subset of $M(S)$ as the dual of $C(S)$ with the strict topology. Hence by $\left[6\right.$, p. 61 , Theorem 6 and p. 46], $\left\{U_{t} \mu: t \geqq 0\right\}$ is a subset of some $\sigma(M, C)$ convex compact set in $M(S)$.

(d) $\Rightarrow$ (a) The proof is essentially like that of [1]. Note that the $\sigma(M, C)$-closure $Q$ of the convex hull of $\left\{U_{t} \mu: t \geqq 0\right\}$ is $\sigma(M, C)$ compact, assigns a measure of at least the number $a \mu(S)>0$ to $S$, and is invariant under all operators $U_{t}, t>0$. By the Markov-Kakutani Theorem [4, p. 456], $\exists \nu \in Q$ such that $U_{t} \nu=\nu$ for all $t \geqq 0$ proving (a).

It is clear that $(\mathrm{a}) \Rightarrow(\mathrm{e})$ while $(\mathrm{e}) \Rightarrow(\mathrm{f})$ by $[5, \mathrm{pp} .341$ and 430$]$. If $(f)$ holds, then by $[\mathbf{5}$, p. 434] the (variation) norm closure of the convex hull of $K$ is $\sigma\left(M(S), M(S)^{*}\right)$-compact and hence also $\sigma(M, C)$ compact. Consequently (d) holds.

Before considering the results in [2] we note that in the light of [4, Theorems 2.1 and 2.2] the above statements are equivalent to: 
(g) $\exists \mu \in M(S)^{+}$such that $\left\{U_{t} \mu: t \geqq 0\right\}$ is tight. That is, given $\epsilon>0 \exists$ a compact $K \subset S$ such that

$$
U_{\iota} \mu(S \backslash K)<\epsilon \text { for all } t \geqq 0,
$$

while, as noted in [8, Section II and Theorem 2] condition (f) is equivalent to

(h) $\exists \mu \in M(S)^{+}$such that for each open set $U \subset S$ and each $\epsilon>0 \exists$ a compact set $K \subset U$ such that $U_{t} \mu(U / K)<\epsilon$ for all $t \geqq 0$.

Finally, Conway has noted in his dissertation that $(\mathrm{g})$ holds if and only if $\exists \mu \in M(S)^{+}$such that every sequence in $\left\{U_{t} \mu: t \geqq 0\right\}$ has a $\sigma(M, C)$ cluster point in $M(S)$ since we are here dealing only with positive measures.

In closing we first note that (g) is similar to condition (iii) in [2]. Secondly, when the integrals mentioned below exist in $M(S)$, we obtain

(k) $\exists \mu \in M(S)^{+}$and $K$ a $\sigma(M, C)$-compact subset of $M(S)$ such that $1 / t_{n} \int_{0}^{l_{n}} U_{s} \mu d s \in K$ for some sequence $t_{n} \rightarrow \infty$ as an analogue of [2, (iv)] readily from (d). One can then use $(k)$ to imply (a), for the sequence $\left\{1 / t_{n} \int_{0}^{t_{n}} U_{s} \mu d s\right\}$ must then have a nonzero $\sigma(M, C)$ cluster point in $M(S)^{+}$which, as in the latter part of the proof of $(\mathrm{v}) \Rightarrow(\mathrm{i})$ in [2], is an invariant measure for $\left\{U_{t}: t>0\right\}$. Thus, as the referee suggested was possible, our main theorem can be obtained as in [2] without the use of the Markov-Kakutani theorem.

\section{REFERENCES}

1. V. E. Beneš, Existence of finite invariant measures for Markov processes, Proc. Amer. Math. Soc. 18 (1967), 1058-1061.

2. - Finite regular invariant measures for Feller processes, J. Appl. Probability 5 (1968), 203-209.

3. R. C. Buck, Bounded continuous functions on a locally compact space, Michigan Math. J. 5 (1958), 95-104.

4. J. B. Conway, The strict topology and compactness in the space of measures, Trans. Amer. Math. Soc. 126 (1967), 474-486.

5. N. Dunford and J. T. Schwartz, Linear operators, Part I, Interscience, New York, 1958.

6. A. P. Robertson and W. J. Robertson, Topological vector spaces, Cambridge Press, London, 1964.

7. F. D. Sentilles, Kernel representations of operators and their adjoints, Pacific J. Math. 23 (1967), 153-162.

8. - Compactness and convergence in the space of measures, Illinois J. Math. (to appear).

9. F. D. Sentilles and D. C. Taylor, Factorizations in Banach algebras and the general strict topology, Trans. Amer. Math. Soc. (to appear).

UNIVERSITY OF MISSOURI 